\title{
Gestational hypertension and preeclampsia after kidney donation: a nationwide population-based cohort study from Korea
}

\author{
Hyung Soon Lee ${ }^{1}$, Juhan Lee², Kyu Ha Huh², So Ra Yoon ${ }^{3}$ \\ ${ }^{1}$ Department of Surgery-Transplantation, National Health Insurance Service Ilsan Hospital, Goyang, Korea
${ }^{2}$ Department of Surgery-Transplantation, Severance Hospital, Seoul, Korea
${ }^{3}$ Research Institute, National Health Insurance Service Ilsan Hospital, Goyang, Korea
}

Background: Living kidney donation has known to increase the risk of gestational hypertension or preeclampsia. However, pregnancy outcomes in Asian living kidney donors are scarce.

Methods: We performed a retrospective cohort study on 112 living kidney donors and 672 healthy non-donors using the Korean National Health Insurance Claims database from 2007 to 2018. Donors and non-donors were matched with respect to age, residency, income, insurance, and number of pregnancies.

Results: Gestational hypertension or preeclampsia was more common in living kidney donors than in non-donors (12 of 112 donors [10.71\%] vs. 37 of 672 non-donors [5.51\%]; odds ratio, 1.99; $95 \%$ confidence interval, $1.04-3.82 ; P=0.03)$. Use of antihypertensive drugs during preeclampsia was also more common in donors than in non-donors ( 3 of 112 donors [2.68\%] vs. 4 of non-donors [0.60\%]; odds ratio, 4.56; $95 \%$ confidence interval, $1.02-20.37 ; \mathrm{P}<0.01)$. There were no significant differences in preterm birth and low birth weight between two groups. There were no reports of maternal death or neonatal death in the donors.

Conclusions: Our findings indicate that living kidney donors appear to be associated with increased risk of gestational hypertension or preeclampsia compared to matched healthy non-donors.

Corresponding author: Hyung Soon Lee

E-mail: crew3236@hanmail.net

\section{(C) The Korean Society for Transplantation}

This is an Open Access article distributed under the terms of the Creative Commons Attribution Non-Commercial License (http://creativecommons.org/licenses/by-nc/4.0/) which permits unrestricted non-commercial use, distribution, and reproduction in any medium, provided the original work is properly cited. 